\title{
Síndrome de Guillain Barré e hidrocefalia en un lactante con Síndrome de Wiskott Aldrich
}

\author{
Guillain-Barré Syndrome and Hydrocephalus in an infant \\ with Wiskott-Aldrich Syndrome
}

\author{
Daniela Ávila-Smirnow ${ }^{\mathrm{a}, \mathrm{b}}$, Marcela Córdova-Aguilera ${ }^{\mathrm{c}}$, Christián Cantillano-Malone ${ }^{\mathrm{d}, \mathrm{e}}$, \\ Manuel Arriaza-Ortiz ${ }^{\mathrm{a}, \mathrm{b}}$, Adriana Wegner-Araya ${ }^{\mathrm{f}}$
}

\begin{abstract}
aUnidad de Neurología, Servicio de Pediatría, Complejo Asistencial Dr. Sótero del Río, Chile
bunidad de Neurología, División de Pediatría, Escuela de Medicina, Pontificia Universidad Católica de Chile ‘unidad de Hematología-Oncología, Servicio de Pediatría, Complejo Asistencial Dr. Sótero del Río, Chile

aUnidad de Cirugía, Servicio de Pediatría, Complejo Asistencial Dr. Sótero del Río, Chile

eUnidad de Neurocirugía, División de Cirugía, Escuela de Medicina, Pontificia Universidad Católica de Chile

fUnidad de Cuidados Intensivos, Servicio de Pediatría, Complejo Asistencial Dr. Sótero del Río, Chile
\end{abstract}

Recibido: 26 de abril de 2019; Aceptado: 27 de agosto de 2019

¿Qué se sabe del tema que trata este estudio?

El Síndrome de Guillain-Barré (SGB) es la neuropatía adquirida más frecuente en pediatría, sin embargo en lactantes menores de un año, sólo existen reportes de casos. Su asociación con el Síndrome de Wiskott-Aldrich (SWA) ha sido presentada en sólo dos niños previamente.
¿Qué aporta este estudio a lo ya conocido?

En este artículo se presenta el tercer caso de un lactante en el que se asocian SWA y SGB, con buena respuesta a inmunoglubulinas intravenosas. Es importante tener un alto grado de sospecha de SGB en lactantes con SWA que presenten parálisis fláccida aguda.

\section{Resumen}

El Síndrome de Guillain-Barré (SGB) es raramente diagnosticado en lactantes menores de 1 año. Su asociación con el Síndrome de Wiskott Aldrich (SWA), es aún menos frecuente, y ha sido previamente reportada sólo en dos pacientes a nuestro conocimiento. La hidrocefalia, es una complicación conocida, pero infrecuente del SGB. Objetivo: presentar el caso clínico de un lactante en el que se asocian las patologías de SGB, SWA e hidrocefalia. Caso Clínico: varon de 9 meses, portador de SWA hospitalizado en unidad de cuidados intensivos por hipotonía aguda y compromiso del estado general. Evolucionó con parálisis fláccida, falla ventilatoria y arreflexia generalizada. Una punción lumbar mostró disociación albuminocitológica, y el estudio electrofisiológico mostró signos de polineuropatía desmielinizante severa. Se trató con inmunoglobulina, evolucionando en forma satisfactoria. Por bradicardia intermitente, se realizó tomografía axial computada cerebral (TAC), que mostró signos
Palabras clave: estudio electrofisiológico; Síndrome de Guillain-Barré; Síndrome de Wiskott-Aldrich; Hidrocefalia; Inmunoglobulina; Trasplante de médula ósea 
de una hidrocefalia aguda, manejada mediante válvula derivativa ventrículo peritoneal con favorable respuesta. En el largo plazo, se sometió a trasplante de médula ósea y debió ser reintervenido por complicaciones valvulares, sin embargo, su desarrollo psicomotor es normal sin secuelas neurológicas evidentes hasta los 3 años. Conclusión: Presentamos el tercer caso de SGB en un paciente portador de SWA, destacando ser el primero de ellos en un lactante menor de 1 año. Adicionalmente, este niño presentó una hidrocefalia aguda como complicación del SGB. Consideramos relevante tener presente estas comorbilidades, debido a que su pronto diagnóstico y manejo oportuno, permiten una mejor recuperación neurológica y evitan complicaciones potencialmente letales.

\section{Keywords:}

Electromyography; Guillain-Barre

Syndrome;

Wiskott-Aldrich

Syndrome;

Immunoglobulins;

Bone Marrow

Transplantation

\section{Introducción}

El SGB es la polirradiculoneuropatía adquirida más frecuente tanto en pacientes pediátricos como adultos, con incidencia de 0,6/100.000 habitantes al año en adultos $^{1} y$ en menores de 10 años $^{2}$. Sin embargo en lactantes menores de 12 meses su incidencia es desconocida, limitándose al reporte de casos ${ }^{3}$. Es un cuadro autoinmune debido a anticuerpos dirigidos a raíces espinales y nervios periféricos, siendo precedido frecuentemente por un cuadro infeccioso ${ }^{4}$. Existen formas desmielinizantes, axonales y variantes.

El SWA es una rara inmunodeficiencia primaria ligada al X, caracterizada por microtrombocitopenia, eczema, infecciones y un mayor riesgo de manifestaciones autoinmunes y neoplasias ${ }^{5,6}$. La incidencia de SWA se ha estimado en menos de 1-10 en 1.000 .000 nacidos vivos masculinos ${ }^{6,7}$. Se debe a mutaciones en el gen Wikott-Aldrich syndrome (WAS), que codifica la proteína del síndrome de Wiskott-Aldrich (WASP), la que participa en procesos de inmunidad adaptativa e innata ${ }^{8}$.

A pesar de que el SWA suele asociarse a trastornos disinmunitarios, sólo existen dos reportes previos de niños que presentaron SGB como comorbilidad aso- ciada $^{9,10}$. A continuación, presentamos un tercer niño en el que se asociaron los SWA, SGB y adicionalmente una hidrocefalia aguda.

\section{Caso Clínico}

\section{Antecedentes perinatales y médico-quirúrgicos:}

Lactante de género masculino, portador de SWA. Desde el punto de vista genético, documentado por la presencia de una variante patogénica en el gen WAS (c.27_28insC, p.Pro10Profs ${ }^{\star} 26$ ). Se trataba con inmunoglobulina $0,5 \mathrm{~g} / \mathrm{kg}$ mensual y transfusiones plaquetarias según requerimiento. Logró sedestación independiente a los 6 meses, y su desarrollo psicomotor fue normal hasta los 8 meses de edad.

\section{Presentación clínica, estudio y manejo iniciales:}

Ingresó a unidad de cuidados intensivos a los 9 meses de vida en forma electiva, para administración de su tratamiento mensual con inmunoglobulina. Sin embargo, destacaba historia de 1 mes de evolución de compromiso del estado general, pérdida de la sedestación, disminución de incremento pondoestatural y de la ingesta alimentaria. En este período consultó a nutriólogo, quien solicitó video deglución y urocultivo 
por sondeo, aumentó aporte calórico y citó a control. Por persistencia de síntomas en el control, se sugirió hospitalizar para realizar estudio y manejo intensivo, el cual fue postergado por falta de camas.

En la evaluación de ingreso a unidad de cuidados intensivos, el paciente se apreciaba en malas condiciones generales, decaído, irritable, deshidratado, con polipnea, taquicardia, y aspecto tóxico, habiendo perdido $230 \mathrm{~g}$ en 5 días previo a la hospitalización. Por su gravedad, se sospechó cuadro séptico, y se inició estudio en el que destacaba hemograma con recuento de blancos de $20.620 / \mathrm{uL}$, proteína $\mathrm{C}$ reactiva de $91 \mathrm{mg} / \mathrm{L}$ e inmunofluorescencia directa viral negativa (virus respiratorio sincicial, adenovirus, parainfluenza 1-2-3 e influenza A-B). Se solicitó hemocultivo, urocultivo, cultivo de líquido cefalorraquídeo y se indicó tratamiento antibiótico de amplio espectro con ceftriaxona y vancomicina, por sospecha de meningitis bacteriana.

El $1^{\text {er }}$ día de hospitalización, se observó mayor compromiso de su condición clínica, destacando al examen neurológico una hipotonía global, pérdida de control cefálico y de la sedestación, con reflejos osteotendíneos presentes, lo que se atribuyó a sepsis en curso. Por deterioro ventilatorio requirió intubación para ventilación mecánica invasiva. Se realizó punción lumbar que mostró líquido claro, pero con presión que impresionó aumentada, sin leucocitos, Gram sin bacterias, glucosa
$70 \mathrm{mg} / 100 \mathrm{ml}$, proteínas 2,27 g/l. Inicialmente, esta hiperproteinorraquia se interpretó como una encefalitis y se solicitó TAC en la que se observó ventrículos de tamaño aumentado a expensas de atrofia cerebral (figura 1-A). Los cultivos solicitados al ingreso, fueron negativos.

Por mejoría en parámetros ventilatorios, se extubó el $4^{\circ}$ día de hospitalización y se suspendió sedación. Sin embargo, evolucionó con mala mecánica ventilatoria, requiriendo reintubación. Paralelamente, se fue instaurando disminución de reflejos osteotendíneos de extremidades superiores y arreflexia de extremidades inferiores, por lo que se repitió punción lumbar. Se observó líquido claro incoloro, glucosa $83 \mathrm{mg} / 100 \mathrm{ml}$, proteínas 2,56 g/l, 3 leucocitos/ml y Gram sin bacterias. En este contexto, se sospechó un SGB, siendo tratado con inmunoglobulinas $1 \mathrm{~g} / \mathrm{kg} /$ día por 2 días.

Por bradicardia intermitente, se realizó control de TAC el $7^{\circ}$ día de hospitalización, que mostró mayor ventriculomegalia y borramiento de surcos, diagnosticándose una hidrocefalia aguda asociada a SGB (figura 1-B). Se instaló drenaje ventricular externo, y al día siguiente, TAC de control mostró drenaje bien ubicado, menor tamaño de ventrículos y mayor presencia de surcos.

Desde el punto de vista ventilatorio, evolucionó favorablemente, extubándose el $9^{\circ}$ día y pasando a BiPAP. Se realizó resonancia magnética de cerebro que
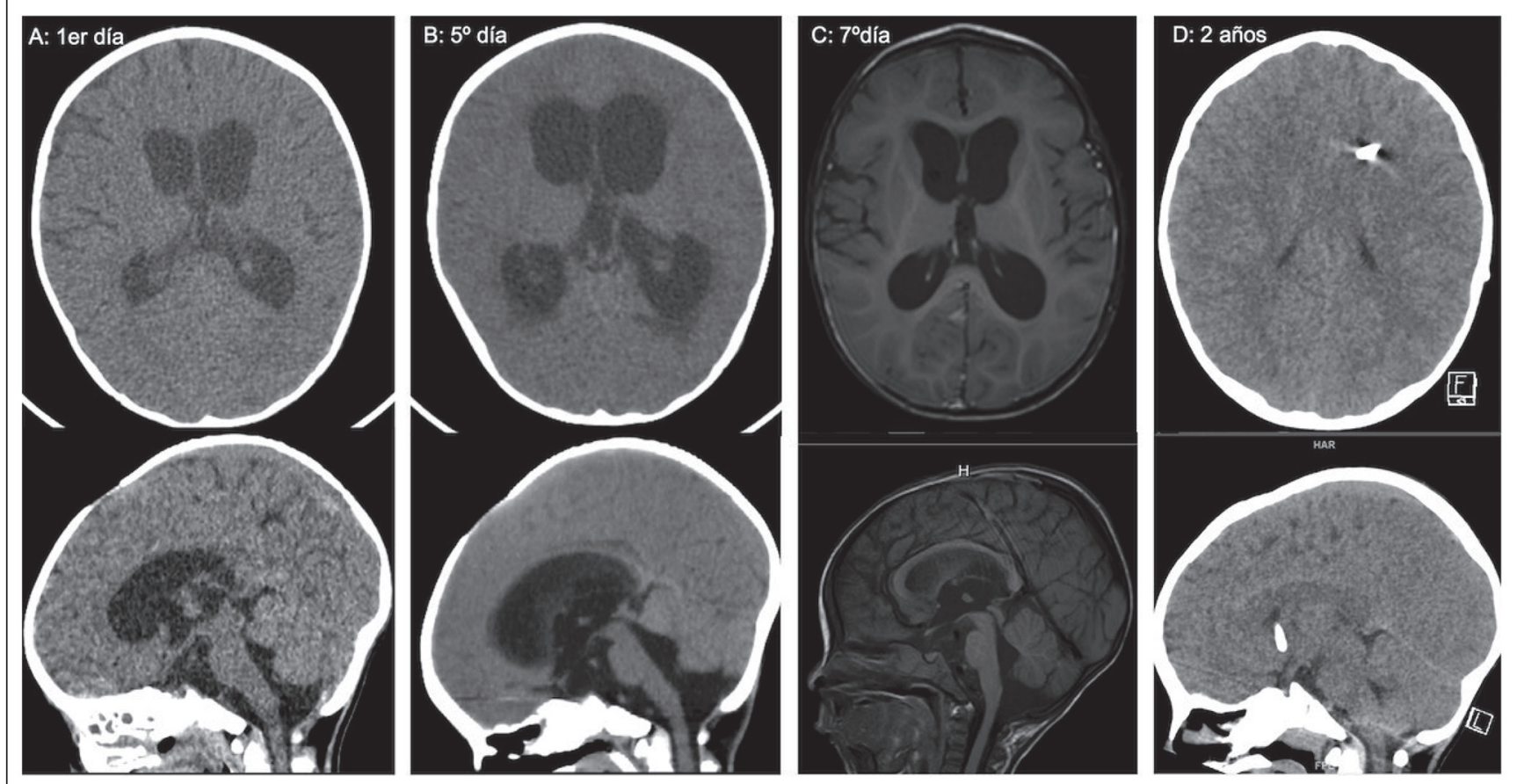

Figura 1. Cortes axiales (arriba) y coronales (abajo) de neuro-imágenes efectuadas. A: TAC al ingreso muestra ventriculomegalia moderada. B: TAC de control en el que se observa aumento de la dilatación ventricular. C: Resonancia magnética posterior a la instalación de válvula derivativa ventricular en la que se observa disminución del volumen ventricular. D: TAC de control a los 2 años de seguimiento, muestra ventrículos de tamaño normal y válvula in situ. 

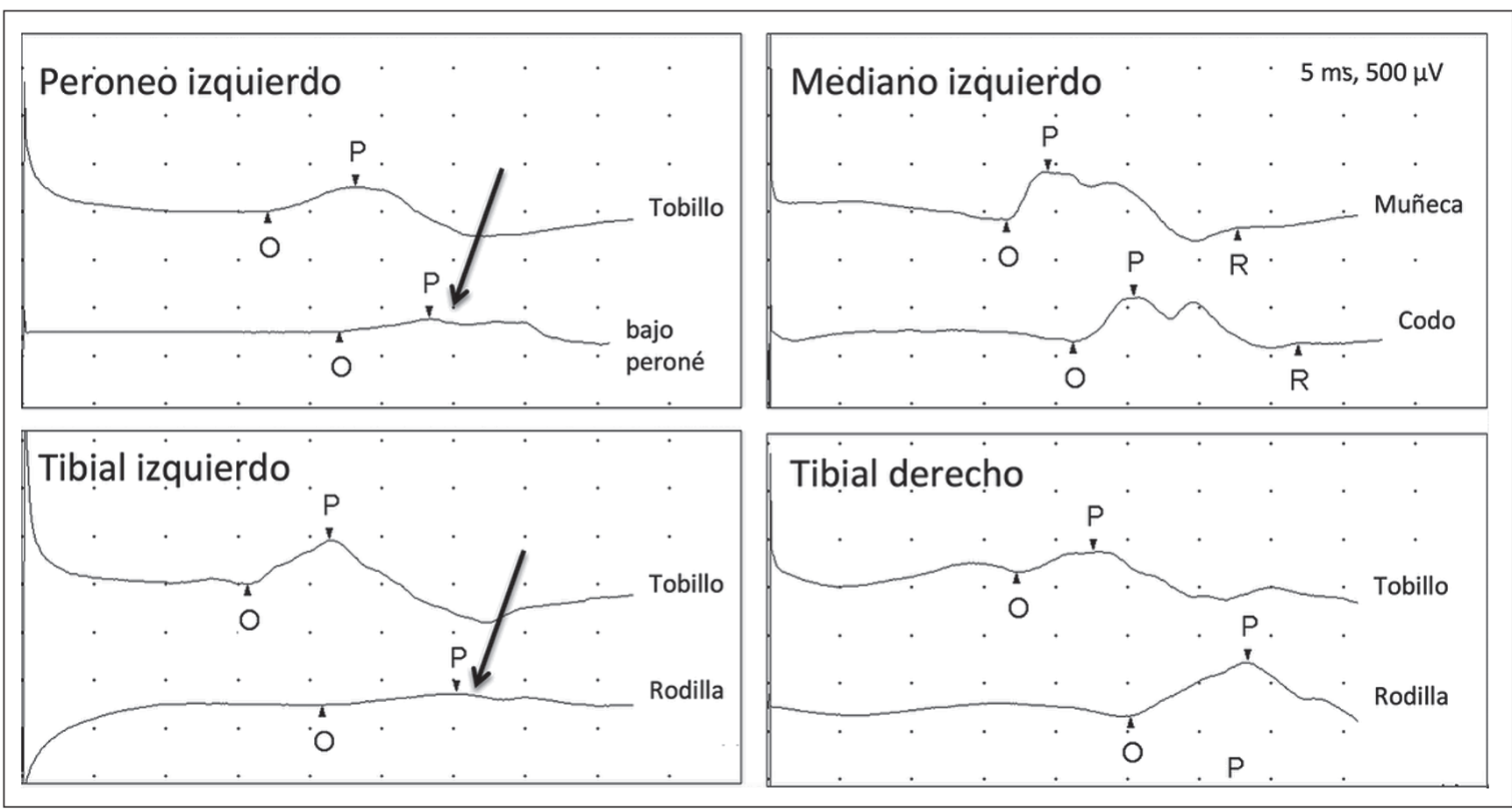

Figura 2. Estudios de neuro-conducción motora de nervios peroneo, tibial y mediano izquierdos, y tibial derecho: se observa aumento de las latencias proximales y disminución de las amplitudes en todos los nervios. Las flechas muestran bloqueo de conducción. O (onset): inicio, P (peak): pico.

Tabla 1. Estudio electrofisiológico

\begin{tabular}{lccc}
\hline Nervio & Latencia proximal $(\mathrm{ms})$ & Amplitud $(\mathrm{mV})$ & Velocidad de conducción $(\mathrm{m} / \mathrm{s})$ \\
\hline Ulnar y mediano derechos & Respuesta ausente & & \\
Mediano izquierdo & 16,6 & $0,5(\mathrm{VN}>1)$ & $16(\mathrm{VN}>34)$ \\
Peroneo izquierdo & 17,1 & $0,3(\mathrm{VN}>1,5)$ & $26(\mathrm{VN}>32)$ \\
Tibial derecho & 17,3 & $0,5(\mathrm{VN}>5)$ & $14(\mathrm{VN}>32)$ \\
Tibial izquierdo & 15,7 & $0,2(\mathrm{VN}>5)$ & $27(\mathrm{VN}>32)$ \\
\hline
\end{tabular}

Se observa marcado aumento de las latencias y disminución de las amplitudes de todos los nervios estudiados. La velocidad de conducción está severamente disminuida en los nervios mediano izquierdo y tibial derecho. ms: milisegundos, mV: milivoltios, m/s: metros por segundo, VN: valor normal.

mostró hidrocefalia derivada sin cambios respecto a último TAC (figura 1-C).

Una vez extubado y sin sedación, se realizó evaluación por neurología el $10^{\circ}$ día de hospitalización, observándose hipotonía severa y debilidad. Sólo lograba movilizar extremidades en el plano sin vencer gravedad y tenía arreflexia global. Se realizó estudio electrofisiológico que mostró poliradiculoneuropatía desmielinizante (figura 2, tabla 1). Por persistencia de marcada debilidad y dependencia de ventilación no invasiva, se repitió dosis de inmunoglobulina con el mismo esquema previo.

\section{Evolución a corto plazo}

Continuó mejoría ventilatoria durante la tercera semana de evolución, suspendiéndose BiPAP y oxí- geno. Se sometió a internalización de derivativa ventricular externa y se controló TAC que mostró menor dilatación ventricular.

La $4^{\text {a }}$ semana comenzó a recuperar potencia muscular, logrando progresivamente vencer gravedad con las 4 extremidades. Reevaluado la $5^{\text {a }}$ semana, lograba vencer gravedad y resistencia, ya se obtenian reflejos osteotendíneos, aunque persistía hipotonía en menor cuantía.

Fue dado de alta luego de un mes y medio de hospitalización, en buenas condiciones generales, con examen neurológico en el que destacaba paciente vigil, bien contectado, lograba seguir con la mirada, pares craneanos normales, con mínima hipotonía de tronco pero sin debilidad, control cefálico completo, pero sin recuperar aún sedestación independiente, reflejos 
osteotendíneos globalmente disminuidos, plantares flexores, sin clonus, ausencia de reflejos de paracaídas lateral y horizontal.

\section{Evolución a largo plazo}

Se realizó trasplante de sangre de cordón umbilical, de donante no emparentado, a los 12 meses de edad y evolucionó sin complicaciones significativas. En dos ocasiones, a los 28 y 37 meses de edad debió ser reintervenido por disfunción valvular, en forma satisfactoria (figura 1-D).

No presentó nuevas intercurrencias de importancia y su desarrollo psicomotor mejoró progresivamente en el curso de los meses siguientes, recuperando sedestación a los 12 meses y adquiriendo marcha a los 18. Su última evaluación neurológica se efectuó a los 3 años 2 meses, destacando un desarrollo psicomotor y un examen motor normales. En esta última consulta se observó que se contactaba adecuadamente, se relacionaba bien con sus pares, comprendía instrucciones complejas, construía frases de hasta 5 palabras, se alimentaba y vestía parcialmente independiente y controlaba esfínteres. Su marcha era normal, corría y subía escaleras. No presentaba síntomas residuales como fatigabilidad, trastornos de la marcha, ni anomalías sensitivas.

\section{Discusión}

Presentamos el caso de un lactante de 9 meses, portador de un SWA, que debutó con una parálisis fláccida aguda, arreflexia, disociación albúmino citológica y hallazgos electrofisiológicos compatibles con una variante desmielinizante de SGB. Este cuadro se asocia a una hidrocefalia aguda, atribuida a una complicación del SGB.

El SGB, es infrecuente en lactantes, especialmente antes del año de edad, sin embargo se han reportado casos desde la edad neonatal ${ }^{11}$. En niños pequeños, la sospecha clínica suele ser más baja que a edades mayores, por su menor frecuencia y porque la historia y exámenes neurológicos son más difíciles de obtener, así como la realización de una EMG por un neurofisiólogo con experiencia en este grupo etáreo ${ }^{12}$. Es por ello, que se ha reportado un importante retraso en el diagnóstico en lactantes pequeños ${ }^{3}$. El tratamiento utilizado en lactantes ha consistido en inmunoglobulina intravenosa en la mayoría de los $\operatorname{casos}^{12,13}$. En un artículo, se describe incluso el uso exitoso de plasmaféresis ${ }^{13}$, sin embargo, la experiencia es muy limitada en este grupo etáreo, su seguridad no ha sido aún dilucidada y por sus potenciales complicaciones, no se recomienda su uso rutinario ${ }^{14}$.

A pesar de tratarse de una inmunodeficiencia, los trastornos autoinmunes son frecuentes en el SWA, en- contrándose entre el 22 y $72 \%$ de los pacientes ${ }^{15}$; se ha descrito la presencia de anemia hemolítica autoinmune, vasculitis, artritis, neutropenia, enfermedad inflamatoria intestinal y nefropatía por IgA ${ }^{15}$. A su vez, se ha reportado la asociación de SGB a infecciones que cursan con inmnodeficiencias como $\operatorname{VIH}^{16} \mathrm{y}$ a cuadros disinmunitarios como artritis reumatoide ${ }^{17}$, Síndrome de Sjögren ${ }^{18}$ y enfermedad celíaca ${ }^{19}$.

En la literatura internacional, se reportan dos preescolares de 2 años portadores SWA en los que se asoció un $\mathrm{SGB}^{9,10}$. Uno de los niños tuvo un SGB de tipo desmielinizante y el segundo de tipo axonal. El primer niño, consultó por dificultad de la marcha y debilidad de extremidades inferiores de una semana de evolución, durante un episodio de tos prolongado, destacando arreflexia al examen neurológico de ingreso, por lo que se sospechó $\mathrm{SGB}^{10}$. El segundo niño, presentó una sepsis con cultivos negativos, requiriendo ventilación mecánica; luego de una semana, a pesar de estabilidad hemodinámica, impresionaba con compromiso de conciencia leve y no seguía instrucciones, sus reflejos osteotendíneos estaban presentes. Inicialmente, se sospechó una encefalopatía, se realizó resonancia magnética encefálica y punción lumbar, destacando disociación albúmino citológica; esto elevó la sospecha de SGB ${ }^{9}$. Ambos casos fueron confirmados por disociación albúminocitológica y estudio electrofisiológico, siendo tratados con inmunogobulina intravenosa, con buena respuesta. Este tercer caso de SGB en un niño portador de SWA, sugiere que existiría una asociación entre ambas patologías, ya que, dada la baja frecuencia de ambas entidades es poco probable observarlas en un mismo paciente, lo que se explicaría por los mecanismos fisiopatológicos subyacentes debidos a los trastornos inmunitarios que comparten ambas pataologías.

El aumento de la proteinorraquia se ha reportado en casos aislados con el uso de inmunoglobulina, siendo secundario a una meningitis aséptica ${ }^{20}$. Pensamos que la hiperpoteinorraquia en este paciente, se explica principalmente por la inflamación radicular propia del SGB. El tratamiento crónico con inmunoglubulina pudo haber tenido un rol, pero mucho menor en la hiperproteinorraquia que presentó.

La asociación de SGB e hidrocefalia aguda, es infrecuente. Se piensa que la etiopatogenia corresponde a una obstrucción a la circulación del líquido cefalorraquídeo debida a hiperproteinorraquia o a disminución de la absorción del mismo ${ }^{21,22}$. En nuestro paciente, la hiperproteinorraquia alcanzó valores muy elevados, por lo que probablemente tuvo un rol fundamental en su etiopatogenia.

La evolución del SGB a largo plazo suele ser favorable en niños, con recuperación completa de la función motora en la mayoría de ellos, como el caso acá presen- 
tado; a pesar de ello las secuelas motoras reportadas, aunque infrecuentes, son relevantes ya que se asocian a discapacidad, incluyendo pérdida de la marcha y diplegia facial ${ }^{23,24}$. Además, se ha reportado la persistencia de síntomas residuales menos severos como parestesias, inestabilidad de la marcha, dolor de pies y manos y fatigabilidad en hasta el 65\% de los niños ${ }^{23}$.

\section{Conclusión}

Presentamos el tercer paciente portador de SWA asociado a un SGB, el que a su vez se complica con una hidrocefalia aguda. Sugerimos tener presente el diagnóstico de SGB en niños con SWA que presenten parálisis flaccida y recordar que la hidroceflia es una complicación en pacientes con SGB. Una pesquiza y manejo precoz de estas complicaciones, permitirán cambiar el pronóstico vital y de neurodesarrollo, como ocurrió en este niño.

\section{Responsabilidades Éticas}

Protección de personas y animales: Los autores declaran que los procedimientos seguidos se conformaron a las normas éticas del comité de experimentación humana responsable y de acuerdo con la Asociación Médica Mundial y la Declaración de Helsinki.

Confidencialidad de los datos: Los autores declaran que han seguido los protocolos de su centro de trabajo sobre la publicación de datos de pacientes.

Derecho a la privacidad y consentimiento informado: Los autores han obtenido el consentimiento informado de los pacientes y/o sujetos referidos en el artículo. Este documento obra en poder del autor de correspondencia.

\section{Conflicto de intereses}

Los autores declaran no tener conflicto de intereses.

\section{Referencias}

1. Cea G, Jara P, Quevedo F. [Clinical features of Guillain-Barré syndrome in 41 patients admitted to a public hospital]. Rev Med Chile 2015;143(2):183-9.

2. Sejvar JJ, Baughman AL, Wise M, Morgan OW. Population incidence of GuillainBarré syndrome: a systematic review and meta-analysis. Neuroepidemiology. 2011;36(2):123-33.

3. Orlik K, Griffin GD. Guillain-Barré in a 10-month-old: diagnostic challenges in a pediatric emergency. Am J Emerg Med. 2014;32(1):110.e5-6.

4. Willison HJ, Jacobs BC, van Doorn PA. Guillain-Barré syndrome. Lancet. 2016;388(10045):717-27.

5. Worth AJ, Thrasher AJ. Current and emerging treatment options for WiskottAldrich syndrome. Expert Rev Clin Immunol. 2015;11(9):1015-32.

6. Wietstruck P MA, Zúñiga C P, Talesnik GE, Méndez RC, Barriga CF. [Hematopoietic stem cell transplantation for patients with WiskottAldrich syndrome]. Rev Med Chile 2007;135(7):917-23.

7. Liu DW, Zhang ZY, Zhao Q, et al. Wiskott-Aldrich syndrome/X-linked thrombocytopenia in China: Clinical characteristic and genotype-phenotype correlation. Pediatr Blood Cancer. 2015;62(9):1601-8.

8. Rivers E, Thrasher AJ. Wiskott-Aldrich syndrome protein: Emerging mechanisms in immunity. Eur J Immunol. 2017;47(11):1857-66.

9. Broomall E, Taylor JM, Peariso K. A
2-Year-Old Boy With Difficulty Waking After Bone Marrow Transplantation. Semin Pediatr Neurol. 2018;26:120-3.

10. Olmez A, Turul T, Tezcan I, Turanli G. Guillain-Barré syndrome in an immunocompromised patient with Wiskott-Aldrich syndrome. Turk J Pediatr. 2008;50(4):412-3.

11. Anastasopoulou S, Lindefeldt M, Bartocci $\mathrm{M}$, Wickström R. Acute inflammatory demyelinating polyradiculoneuropathy in a newborn infant. Eur J Paediatr Neurol. 2016;20(5):754-7.

12. Vedrenne-Cloquet M, Maincent $\mathrm{K}$, Billette de Villemeur T, Mayer M. [Guillain-Barré syndrome in infancy: The importance of electroneuromyography]. Arch Pediatr. 2016;23(2):184-7.

13. Akarcan SE, İşgüder R, Yılmaz Ü, Ayhan Y, Ceylan G, Ağın H. GuillainBarre syndrome in a 7-month-old boy successfully applied plasma exchange. Transfus Apher Sci. 2016;54(1):139-43.

14. Tekgunduz SA, Kara A, Bozkaya IO, Cagli A, Ozbek NY. Therapeutic plasma exchange in non-hematooncological disorders in pediatrics: A single center experience. Transfus Apher Sci. 2018;57(1):20-2.

15. Catucci M, Castiello MC, Pala F, Bosticardo M, Villa A. Autoimmunity in wiskott-Aldrich syndrome: an unsolved enigma. Front Immunol. 2012;3:209.

16. Prior DE, Song N, Cohen JA Neuromuscular diseases associated with Human Immunodeficiency Virus infection. J Neurol Sci. 2018;387:27-36.

17. Silburn S, McIvor E, McEntegart A, Wilson H. Guillain-Barré syndrome in a patient receiving anti-tumour necrosis factor alpha for rheumatoid arthritis: a case report and discussion of literature. Ann Rheum Dis. 2008;67(4):575-6.

18. Tanaka K, Nakayasu H, Suto Y, et al. Acute Motor-dominant Polyneuropathy as Guillain-Barré Syndrome and Multiple Mononeuropathies in a Patient with Sjögren's Syndrome. Intern Med. 2016;55(18):2717-22.

19. Pacitto A, Paglino A, Di Genova L, et al. Celiac Disease Presenting with Peripheral Neuropathy in Children: A Case Report. Int J Environ Res Public Health. 2017;14(7).

20. Berg R, Fuellenhals E. Aseptic meningitis following therapy with immune globulins: a combination of product features and patient characteristics? Transfusion. 2016;56(12):3021-8

21. Ozdemir O, Calisaneller T, Sonmez E, Caner H, Altinors N. Atypical presentation of Guillain-Barre syndrome with acute hydrocephalus. Acta Neurochir (Wien). 2008;150(1):87-8; discussion 8.

22. Liu CY, Kao CD, Chen JT, Yeh YS, Wu ZA, Liao KK. Hydrocephalus associated with Guillain-Barre syndrome. J Clin Neurosci. 2006;13(8):866-9.

23. Roodbol J, de Wit MC, Aarsen FK, Catsman-Berrevoets CE, Jacobs BC. Long-term outcome of Guillain-Barré syndrome in children. J Peripher Nerv Syst. 2014;19(2):121-6.

24. Salehiomran MR, Nikkhah A, Mahdavi M. Prognosis of Guillain-Barré Syndrome in Children. Iran J Child Neurol. 2016;10(2):38-41. 